Review

Inflammation and breast cancer

\title{
Microenvironmental factors regulating macrophage function in breast tumours: hypoxia and angiopoietin-2
}

\author{
Claire E Lewis and Russell Hughes
}

Tumour Targeting Group, Academic Unit of Pathology, Section of Infection, Inflammation and Immunity, The Sir Henry Wellcome Laboratories for Medical Research, University of Sheffield Medical School, Beech Hill Road, Sheffield S10 2RX, UK

Corresponding author: Claire E Lewis, claire.lewis@sheffield.ac.uk

Published: 15 June 2007

This article is online at http://breast-cancer-research.com/content/9/3/209

(c) 2007 BioMed Central Ltd

Breast Cancer Research 2007, 9:209 (doi:10.1186/bcr1679)

\begin{abstract}
Considerable evidence has now accumulated for tumour-associated macrophages stimulating key aspects of tumour progression, including the proliferation, survival and metastasis of tumour cells, tumour angiogenesis and suppression of the anti-tumour functions of other immune effectors at the tumour site. Tumour microenvironmental factors such as hypoxia have profound, direct effects on these cells, stimulating many of their pro-tumour functions. Hypoxia also does so indirectly by stimulating the release of the cytokine angiopoietin-2 from tumour cells and tumour blood vessels. This in turn then recruits Tie-2-expressing monocytes into tumours from the bloodstream and inhibits their production of anti-apoptotic and anti-angiogenic cytokines.
\end{abstract}

\section{Role of tumour-associated macrophages in tumour progression}

Two different approaches - the use of various transgenic mouse models and the analysis of human tumours - have demonstrated a close link between the activity of tumourassociated macrophages (TAMs) and tumour progression $[1,2]$. TAMs are abundant in most forms of solid tumour, where they often display a relatively immature phenotype and are positively correlated with tumour angiogenesis and/or progression (reviewed in [3]). Pollard's group crossed PyMTMMTV mice (which spontaneously develop mammary tumours) with the transgenic op/op mouse model lacking the gene for colony-stimulating factor-1, a crucial growth factor for macrophages and their precursors from the bone marrow, namely blood monocytes. The tumours that developed in these macrophage-depleted mice showed a slower rate of progression to malignancy and formed far fewer metastases in the lungs than those in non-macrophage-depleted mice [1]. Moreover, Pollard's group recently characterised the development of the vasculature in PyMT-MMTV tumours during progression to malignancy and showed that the onset of the 'angiogenic switch' (the formation of the high-density vessel network associated with the transition to malignancy) was regulated by TAMs. Preinvasive mammary lesions in op/op mice exhibited both a delayed angiogenic switch and transition to malignancy, whereas genetic restoration of the macrophage population in tumours reversed this [4]. Although these studies suggest that TAMs have a key role in promoting tumour angiogenesis, progression to malignancy and metastasis, they have yet to be confirmed in similar studies with other macrophage-depleted, transgenic mouse tumour models.

However, these data accord well with our finding that high numbers of TAMs correlate with increased tumour angiogenesis, lymph node status and reduced survival of breast cancer patients [5]. Moreover, we showed that TAMs in breast carcinomas express numerous tumour-promoting factors such as the important mitogen epidermal growth factor [6] and the pro-angiogenic cytokine vascular endothelial growth factor (VEGF) [7]. TAMs have also been shown to release a variety of other cytokines and enzymes known to promote tumour invasion, angiogenesis and metastasis $[3,8]$. Recent studies indicate that when macrophages migrate into tumours they downregulate their expression of the potent anti-angiogenic cytokine IL-12 [9].

These findings have prompted investigations into how the tumour microenvironment 'educates' macrophages to perform these pro-tumour activities. Here we outline the important role of tumour hypoxia in this, both in the form of a direct effect on the expression of pro-tumour genes by TAMs, and indirectly by upregulating the pro-angiogenic cytokine angiopoietin-2 (Ang-2), which in turn has profound effects on TAM function.

Ang = angiopoietin; IL = interleukin; TAM = tumour-associated macrophage; TNF = tumour necrosis factor; VEGF $=$ vascular endothelial growth factor. 


\section{Effect of tumour hypoxia on tumour-associated macrophages}

The vasculature in tumours is often disorganised, chaotic and prone to collapse. This results in the formation of multiple areas of inadequate vascular perfusion and hypoxia in solid tumours [10]. For some time it has been known that hypoxia alters the function of tumour cells, stimulating them to release pro-angiogenic factors, de-differentiate, become resistant to most forms of chemotherapy and metastasise [11]. We and others have shown that hypoxia also has marked effects on macrophage function in tumours.

A subpopulation of TAMs gather in hypoxic, avascular and/or necrotic sites in breast tumours $[5,7,12]$, possibly as a result of the release of such macrophage chemoattractants as endothelial-monocyte-activating polypeptide (EMAP) II, endothelin-2 and VEGF by tumour cells in these sites (reviewed in [13]). Furthermore, because macrophages are phagocytes they may also be attracted into hypoxic, perinecrotic areas along a trail of necrotic debris emanating from these areas. Indeed, we have shown recently that necrotic debris generated from the repeated freezing and thawing of a human breast tumour cell line (T47D) acts as a powerful chemoattractant for human macrophages in vitro and that this was mediated in part by their detection of necrotic debris by cell surface receptors called class $A$ scavenger receptors $(R$ Hughes, C Murdoch, S Tazzyman and CE Lewis, unpublished observations). Once they reach a hypoxic area, it seems from the work of Balkwill and colleagues [14] that hypoxia then inhibits macrophage migration, immobilising them in these areas. The interplay of these microenvironmental cues on TAM migration is illustrated in Figure 1.

Exposure to hypoxia in these sites stimulates TAMs to acquire a pro-angiogenic phenotype. For example, it stimulates them to express VEGF [7] and the pro-invasive and pro-angiogenic enzyme matrix metalloproteinase-7 [15], as demonstrated in vitro and in hypoxic areas of breast tumours. Furthermore, we recently demonstrated that when human macrophages infiltrate into the hypoxic centre of human breast tumour spheroids in vitro, they release VEGF and significantly enhance the angiogenic potential of spheroids when they are subsequently implanted into dorsal skin window chambers in nude mice and observed after 3 days [16]. This accords with the finding that hypoxia stimulates primary human macrophages to upregulate more than 30 other pro-angiogenic genes [17]. The impressive array of pro-tumour cytokines, enzymes and cell surface receptors expressed by macrophages in hypoxia are summarised in Figure 2 and reviewed in [18].

Hypoxia-induced gene expression in tumour cells is known to involve the nuclear accumulation of the transcription factors hypoxia-inducible transcription factor (HIF)-1 and HIF-2, which bind to cognate binding sequences in or near the promoters of target genes. Macrophages upregulate both
Figure 1

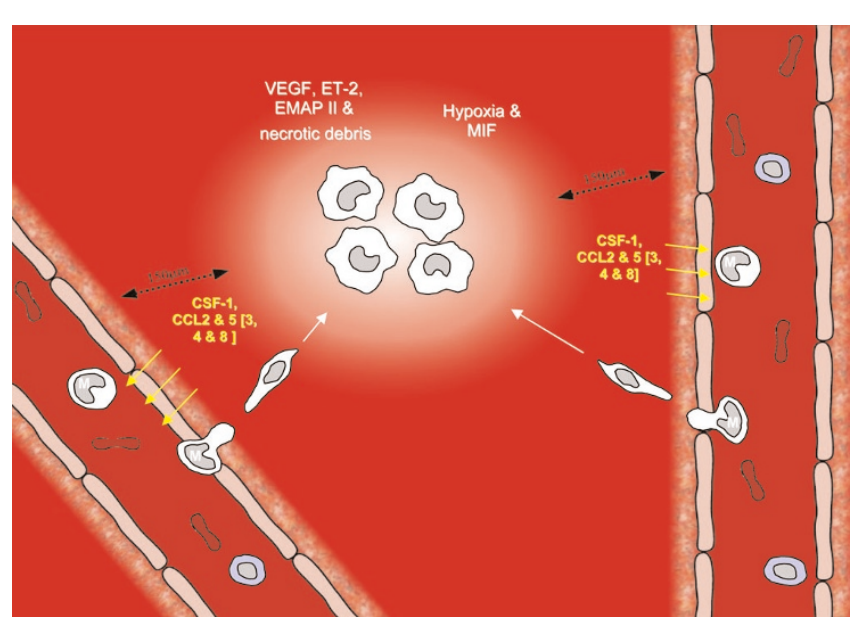

Mechanisms responsible for the accumulation of tumour-associated macrophages in hypoxic areas of solid tumours. A microenronment within a human tumour is shown containing two blood vessels and a hypoxic area (white) that has formed because it is more than 100 to $150 \mu \mathrm{m}$ from either vessel - the critical distance for oxygenation in such tissues. Monocytes $(\mathrm{M})$ pass through these vessels and are recruited into tumours by the release of the monocyte chemoattractants CCL2, 3, 4, 5 and 8 and colony-stimulating factor- 1 (CSF-1) by the tumour. Once monocytes have moved across the tumour vasculature, many are attracted into hypoxic areas by the hypoxia-induced release of other monocyte attractants such as vascular endothelial growth factor (VEGF), endothelin-2 (ET-2) and endothelial-monocyte-activating polypeptide (EMAP) II. These innate cells may also be attracted into sites experiencing chronic hypoxia (and thus cell death) along a trail of necrotic debris emanating from these areas. Hypoxia then acts directly on macrophages to immobilise them and also via the upregulation of macrophage migration inhibitory factor (MIF) by tumour cells, which has a similar effect on macrophage migration (reviewed in [13]).

HIFs when exposed to hypoxia in vitro or inside human tumours $[15,19]$. Furthermore, high expression of one of these (HIF-2) in TAMs in breast carcinomas was correlated with increased tumour vascularity, presumably because of the upregulation by these cells of HIF target genes such as that encoding the pro-angiogenic cytokine VEGF [20]. However, the effects of hypoxia on TAMs are linked to the differentiation status of macrophages: our studies indicate that, unlike fully differentiated macrophages, their precursors, human monocytes from peripheral blood, fail to upregulate either HIF in tumour levels of hypoxia. Rather, they upregulate other hypoxia-induced transcription factors such as Ets-1, ATF-4 (activating transcription factor-4), Egr-1 (early growth response-1), C/EBP $\beta$ (CCAAT-enhancer-binding protein $\beta$ ) and nuclear factor (NF)- $\mathrm{\kappa B}$ [21]. This finding was confirmed by a recent report showing that a human monocytic cell line required exposure to a chemical inducer of differentiation before it could upregulate HIF-1 in response to hypoxia [22]. Because several recent studies have shown that TAMs exhibit a relatively immature phenotype in tumours [23], the exact contribution of these various transcription factors to the 
Figure 2

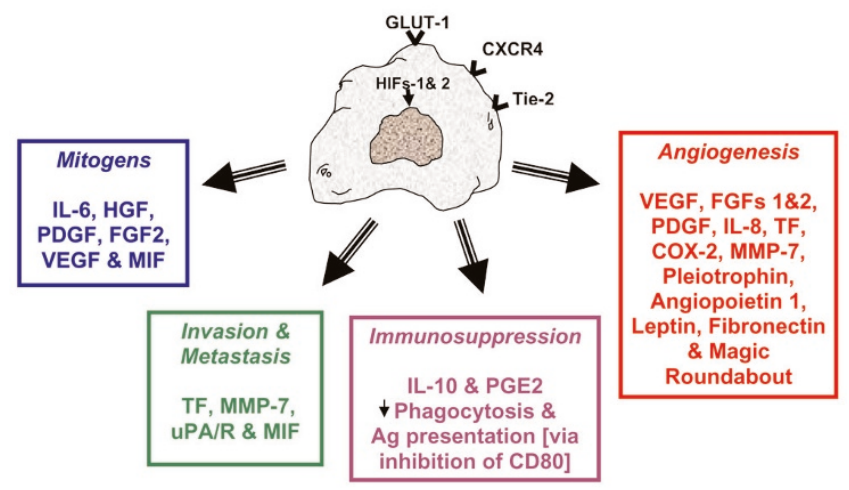

Hypoxia induces marked changes in the phenotype of macrophages. Macrophages upregulate hypoxia-inducible transcription factor (HIF)-1 and HIF-2 in hypoxia, which translocate to the nucleus to induce the expression of a wide array of target genes. Several important cellsurface receptors are upregulated in hypoxia, including the glucose receptor GLUT-1 (for increased glucose uptake as the cell switches to anaerobic glycolysis to make ATP in the absence of oxygen), the chemokine stromal cell-derived factor-1 (SDF-1) receptor CXCR4, and the angiopoietin receptor Tie-2. Hypoxia also stimulates the expression of a wide array of other pro-tumour cytokines, enzymes and receptors, grouped here according to their known function in tumours.

Downregulation of a factor or tumour-associated macrophage function is indicated by an arrow $[15,17,18]$. Ag, antigen; COX, cyclooxygenase; FGF, fibroblast growth factor; HGF, hepatocyte growth factor; MIF, macrophage migration inhibitory factor; MMP, matrix metalloproteinase; PDGF, platelet-derived growth factor; PGE2, prostaglandin $\mathrm{E}_{2} ; \mathrm{TF}$, tissue factor; uPA/R, urokinase plasminogen activator receptor; VEGF, vascular endothelial growth factor.

pro-angiogenic phenotype of hypoxic TAMs now merits further investigation.

\section{Angiopietin-2 and tumour-associated macrophage functions}

The cytokines Ang-1 and Ang-2 regulate processes such as angiogenesis by binding specifically to the receptor tyrosine kinase Tie2/Tek on endothelial cells. Ang-1 acts as a Tie-2 agonist to promote and stabilise mature vessels by promoting interactions between endothelial cells, pericytes, basement membrane and surrounding extracellular matrix. Conversely, Ang-2, the predominant form of angiopoietin in malignant tissues, has been shown to be a functional antagonist of Ang-1 and binds competitively to Tie-2, antagonising the stabilising effect of Ang-1, resulting in an overall destabilisation of existing vessels (reviewed in [24]). In the presence of VEGF these destabilised vessels undergo angiogenic changes and sprout to form new vessels. Thus, angiogenesis is controlled by a dynamic balance between vessel stabilisation and growth, mediated by VEGF, Ang-1 and Ang-2. It has been shown that breast carcinomas express higher levels of Ang-2 than of Ang-1 and that this is correlated with high levels of VEGF expression and tumour angiogenesis [25].
However, Ang-2 has recently been shown to have agonistic functions and to be capable of activating Tie-2 to stimulate endothelial cell migration and tubule formation in vitro [26]. Thus, Ang-2 seems to possess both agonist and antagonist functions when acting on endothelial cells.

Until recently, expression of Tie-2 was thought to be restricted to endothelial cells, but in 2005 De Palma and colleagues showed that a subpopulation of murine blood monocytes expressing Tie-2 are recruited into spontaneous murine and orthotopic human xenograft tumours and have a crucial role in stimulating tumour angiogenesis [27]. We and De Palma's group have now extended these studies to show that $\mathrm{Tie}-2^{+}$monocytes are also abundant in human peripheral blood and exist in a range of human tumours [28,29]. Moreover, Ang-2 was seen in both studies to act as a powerful chemoattractant for these Tie-2 ${ }^{+}$monocytes in vitro and is therefore highly likely to recruit Tie- $2^{+}$monocytes from the bloodstream into tumours $[28,29]$.

Our studies also demonstrated that hypoxia stimulates Tie-2 expression by human monocytes and macrophages [28], suggesting that hypoxia may modulate the response of these cells to Ang-2. Interestingly, several recent studies have shown Ang-2 to be upregulated by tumour cells in hypoxic areas of human tumours [30]. This means that it is highly likely that TAMs would be exposed to both hypoxia and Ang-2 in such areas. This is important because we found that exposure to hypoxia and Ang-2 had marked inhibitory effects on the release of $\mathrm{IL}-12$ by human Tie- $2^{+}$monocytes. This suggests that when monocytes are recruited into tumours and exposed to both Ang-2 and hypoxia it inhibits their ability to mount an anti-angiogenic response. This, together with their hypoxia-induced pro-angiogenic functions, would ensure rapid angiogenesis in (and hence re-oxygenation of) the avascular, hypoxic site.

Moreover, the combined action of Ang-2 and hypoxia also inhibited the release of TNF- $\alpha$ by such cells [29]. This is important because high-dose TNF- $\alpha$ is known to promote the apoptosis of both tumour and endothelial cells [31], so its downregulation near newly formed angiogenic blood vessels could enhance tumour and endothelial cell survival and thus promote metastasis and angiogenesis, respectively. Furthermore, high concentrations of TNF- $\alpha$ inhibit Ang-2 synthesis by endothelial cells [32], so our data suggest that this response of TAMs to Ang-2 may contribute to the high levels of Ang-2 reported in breast tumours [25].

\section{Conclusion}

There is now undeniable evidence that macrophages drive tumour angiogenesis and progression in certain murine mammary tumour models, and this correlates well with studies of their function in human breast tumours. Many TAMs are found in hypoxic areas of such tissues where unequivocal evidence has now been provided to show that 
This article is part of a review series on Inflammation and breast cancer, edited by Mina J Bissell and Jeffrey W Pollard.

Other articles in the series can be found online at http://breast-cancer-research.com/articles/ review-series.asp?series=bcr_Inflammation

hypoxia has profound effects on their function, stimulating them to produce a wide array of pro-tumour growth factors, cytokines and enzymes. Furthermore, hypoxia-induced cytokines such as Ang-2 produced with the tumour microenvironment seem to amplify the effects of hypoxia on TAMs. It is hoped that present attempts to unearth the signalling pathways mediating the powerful effects of hypoxia and Ang-2 on these cells will highlight new targets for new anti-cancer strategies.

\section{Competing interests}

The authors declare that they have no competing interests.

\section{Acknowledgements}

We acknowledge grant support from Yorkshire Cancer Research, UK, and the Biotechnology and Biological Sciences Research Council, UK, for their work in this area.

\section{References}

1. Lin EY, Nguyen AV, Russell RG, Pollard JW: Colony-stimulating factor 1 promotes progression of mammary tumors to malignancy. J Exp Med 2001, 193:727-740.

2. Bingle L, Brown NJ, Lewis CE: The role of tumour-associated macrophages in tumour progression: implications for new anticancer therapies. J Pathol 2002, 196:254-265.

3. Lewis CE, Pollard JW: Distinct role of macrophages in different tumor microenvironments. Cancer Res 2006, 66:605-612.

4. Lin EY, Li JF, Gnatovskiy L, Deng Y, Zhu L, Grzesik DA, Qian H, Xue XN, Pollard JW: Macrophages regulate the angiogenic switch in a mouse model of breast cancer. Cancer Res 2006, 66:11238-11246.

5. Leek RD, Lewis CE, Whitehouse R, Greenall M, Clarke J, Harris AL: Association of macrophage infiltration with angiogenesis and prognosis in invasive breast carcinoma. Cancer Res 1996, 56:4625-4629.

6. O'Sullivan C, Lewis CE, Harris AL, McGee JO: Secretion of epidermal growth factor by macrophages associated with breast carcinoma. Lancet 1993, 342:148-149.

7. Lewis JS, Landers RJ, Underwood JC, Harris AL, Lewis CE: Expression of vascular endothelial growth factor by macrophages is up-regulated in poorly vascularized areas of breast carcinomas. J. Pathol 2000, 192:150-158.

8. Mantovani A, Schioppa T, Porta C, Allavena P, Sica A: Role of tumor-associated macrophages in tumor progression and invasion. Cancer Metastasis Rev 2006, 25:315-322.

9. Sica A, Saccani A, Bottazzi B, Polentarutti N, Vecchi A, van Damme J, Mantovani A: Autocrine production of IL-10 mediates defective IL-12 production and NF-KB activation in tumorassociated macrophages. J Immuno/ 2000, 164:762-767.

10. Vaupel P, Schlenger K, Knoop C, Hockel M: Oxygenation of human tumors: evaluation of tissue oxygen distribution in breast cancers by computerized $\mathrm{O}_{2}$ tension measurements. Cancer Res 1991, 51:3316-3322.

11. Holmquist L, Lofstedt T, Pahlman S: Effect of hypoxia on the tumor phenotype: the neuroblastoma and breast cancer models. Adv Exp Med Biol 2006, 587:179-193.

12. Leek RD, Landers RJ, Harris AL, Lewis CE: Necrosis correlates with high vascular density and focal macrophage infiltration in invasive carcinoma of the breast. Br J Cancer 1999, 79:991-995.
13. Murdoch C, Giannoudis A, Lewis CE: Mechanisms regulating the recruitment of macrophages into hypoxic areas of tumors and other ischemic tissues. Blood 2004, 104:2224-2234.

14. Turner L, Scotton C, Negus R, Balkwill F: Hypoxia inhibits macrophage migration. Eur J Immunol 1999, 29:2280-2287.

15. Burke B, Giannoudis A, Corke KP, Gill D, Wells M, Ziegler-Heitbrock L, Lewis CE: Hypoxia-induced gene expression in human macrophages: implications for ischemic tissues and hypoxia-regulated gene therapy. Am J Pathol 2003,163:12331243.

16. Bingle L, Lewis CE, Corke KP, Reed MW, Brown NJ: Macrophages promote angiogenesis in human breast tumour spheroids in vivo. Br J Cancer 2006, 94:101-107.

17. White JR, Harris RA, Lee SR, Craigon MH, Binley K, Price $T$, Beard GL, Mundy CR, Naylor S: Genetic amplification of the transcriptional response to hypoxia as a novel means of identifying regulators of angiogenesis. Genomics 2004, 83:1-8.

18. Lewis $C$, Murdoch C: Macrophage responses to hypoxia: implications for tumor progression and anti-cancer therapies. Am J Pathol 2005, 167:627-635.

19. Burke B, Tang N, Corke KP, Tazzyman D, Ameri K, Wells M, Lewis CE: Expression of HIF- $1 \alpha$ by human macrophages: implications for the use of macrophages in hypoxia-regulated cancer gene therapy. J Pathol 2002, 196:204-212.

20. Leek RD, Talks KL, Pezzella F, Turley H, Campo L, Brown NS, Bicknell R, Taylor M, Gatter KC, Harris AL: Relation of hypoxiainducible factor- $2 \alpha$ (HIF-2 $\alpha$ ) expression in tumor-infiltrative macrophages to tumor angiogenesis and the oxidative thymidine phosphorylase pathway in human breast cancer. Cancer Res 2002, 62:1326-1329.

21 Elbarghati L, Murdoch C, Lewis CE: Expression of hypoxiainducible transcription factors by human macrophages: involvement of HIF-1 in the hypoxic induction of VEGF and mmP-7. J Leukoc Bio/ 2005, Suppl:19-79.

22. Oda T, Hirota K, Nishi K, Takabuchi S, Oda S, Yamada H, Arai T, Fukuda K, Kita T, Adachi T, et al:: Activation of hypoxiainducible factor 1 during macrophage differentiation. $\mathrm{Am} J$ Physiol Cell Physiol 2006, 291:C104-C113.

23. Konur A, Kreutz M, Knuchel R, Krause SW, Andreesen R: Threedimensional co-culture of human monocytes and macrophages with tumor cells: analysis of macrophage differentiation and activation. Int J Cancer 1996, 66:645-652.

24. Tait CR, Jones PF: Angiopoietins in tumours: the angiogenic switch. J Pathol 2004, 204:1-10.

25. Tsutsui S, Inoue H, Yasuda K, Suzuki K, Takeuchi H, Nishizaki T, Higashi H, Era S, Mori M: Angiopoietin 2 expression in invasive ductal carcinoma of the breast: its relationship to the VEGF expression and microvessel density. Breast Cancer Res Treat 2006, 98:261-266.

26. Kuliszewska K, Maisonpierre PC, Jones N, Campbell Al, Master Z, Bendeck MP, Alitalo K, Dumont DJ, Yancopoulos GD, Stewart DJ: Biological action of angiopoietin-2 in a fibrin matrix model of angiogenesis is associated with activation of Tie2. Cardiovasc Res 2001, 49: 659-670.

27. De Palma M, Venneri MA, Galli R, Sergi Sergi L, Politi LS, Sampaolesi M, Naldini L: Tie2 identifies a hematopoietic lineage of proangiogenic monocytes required for tumor vessel formation and a mesenchymal population of pericyte progenitors. Cancer Cell 2005, 8:211-226.

28. Venneri MA, De Palma M, Ponzoni M, Pucci F, Scielzo C, Zonari E, Mazzieri R, Doglioni C, Naldini L: Identification of pro-angiogenic TIE2-expressing monocytes (TEMs) in human peripheral blood and cancer. Blood in press.

29. Murdoch C, Tazzyman S, Webster S, Lewis CE: Expression of Tie- 2 by human monocytes and their responses to angiopoietin-2. J Immuno/ 2007, 178:7405-7411.

30. Krikun G, F Schatz T, Finlay S, Kadner A, Mesia R, Gerrets H, Lockwood CJ: Expression of angiopoietin-2 by human endometrial endothelial cells: regulation by hypoxia and inflammation. Biochem Biophys Res Commun 2000, 275:159163.

31. Balkwill FR: Tumour necrosis factor and cancer. Prog Growth Factor Res 1992, 14:121-137.

32. Kim I, Kim JH, Ryu YS, Liu M, Koh GY: Tumor necrosis factor- $\alpha$ up-regulates angiopoietin-2 in human umbilical vein endothelial cells. Biochem Biophys Res Commun 2006, 269:361-365. 